\title{
Super Connectivity of Line Graphs and Digraphs
}

\author{
Min L $\ddot{u}^{1}$, Jun-Ming $\mathrm{Xu}^{2}$ \\ ${ }^{1}$ Department of Computer Science and Technology, University of Science and Technology of China, Hefei 230026, \\ China (E-mail: lvmin05@ustc.edu.cn) \\ ${ }^{2}$ Department of Mathematics, University of Science and Technology of China, Hefei 230026, China \\ (E-mail: xujm@ustc.edu.cn)
}

\begin{abstract}
The $h$-super connectivity $\kappa_{h}$ and the $h$-super edge-connectivity $\lambda_{h}$ are more refined network reliability indices than the connectivity and the edge-connectivity. This paper shows that for a connected balanced digraph $D$ and its line digraph $L$, if $D$ is optimally super edge-connected, then $\kappa_{1}(L)=2 \lambda_{1}(D)$, and that for a connected graph $G$ and its line graph $L$, if one of $\kappa_{1}(L)$ and $\lambda_{2}(G)$ exists, then $\kappa_{1}(L)=\lambda_{2}(G)$. This paper determines that $\kappa_{1}(B(d, n))$ is equal to $4 d-8$ for $n=2$ and $d \geq 4$, and to $4 d-4$ for $n \geq 3$ and $d \geq 3$, and that $\kappa_{1}(K(d, n))$ is equal to $4 d-4$ for $d \geq 2$ and $n \geq 2$ except $K(2,2)$. It then follows that $B(d, n)$ and $K(d, n)$ are both super connected for any $d \geq 2$ and $n \geq 1$.
\end{abstract}

Keywords Line graphs, super connectivity, super edge-connectivity, de Bruijn digraphs, Kautz digraphs 2000 MR Subject Classification 05C40

\section{Introduction}

It is well-known that when the underlying topology of an interconnection network is modelled by a connected graph or strongly connected digraph $G=(V, E)$, where $V$ is the set of processors and $E$ is the set of communication links in the network, the connectivity $\kappa(G)$ or the edgeconnectivity $\lambda(G)$ of $G$ is an important measurement for fault-tolerance of the network. In general, the larger $\kappa(G)$ or $\lambda(G)$ is, the more reliable the network is. It is well known that $\kappa(G) \leq \lambda(G) \leq \delta(G)$, where $\delta(G)$ is the minimum degree of $G$. A graph (digraph) $G$ is called to be maximally connected if $\kappa(G)=\delta(G)$ and maximally edge-connected if $\lambda(G)=\delta(G)$. One might be interested in more refined indices of reliability. As more refined indices than the connectivity and the edge-connectivity, the super connectivity and the super edge-connectivity were proposed in $[1,2]$. A graph (digraph) $G$ is super connected if every minimum vertex-cut isolates a vertex of $G$. A super edge-connected graph (digraph) is similarly defined. Since then it has been found that many well-known graphs are super connected or super edge-connected. In particular, Soneoka ${ }^{[10]}$ showed that the de Bruijn digraph $B(d, n)$ is super edge-connected for any $d \geq 2$ and $n \geq 1$; Fábrega and Fiol ${ }^{[5]}$ proved that the Kautz digraph $K(d, n)$ is super edge-connected for any $d \geq 3$ and $n \geq 2$.

A quite natural problem is that if a (strongly) connected (di)graph $G$ is super connected or super edge-connected, then how many vertices or edges must be removed to disconnect $G$ such that every (strongly) connected component of the resulting graph contains no isolated vertices. This problem results in the concept of the super (edge-) connectivity, introduced in [6].

Manuscript received January 5, 2004.

Supported by the National Natural Science Foundation of China (No.10271114, No.10301031). 
For a given nonnegative integer $h$, a vertex-set $S$ of $G$ is called an $h$-super vertex-cut if $G-S$ is not (strongly) connected and every (strongly) connected component contains at least $(h+1)$ vertices. In general, $h$-super vertex-cuts do not always exist. The $h$-super connectivity $\kappa_{h}(G)$ is the minimum cardinality of an $h$-super vertex-cut in $G$ if $h$-super vertex-cuts exist, and, by convention, is $\infty$ otherwise. It is clear that $\kappa_{0}(G)=\kappa(G)$. It is easy to see if $\kappa_{1}(G)>\kappa(G)$ then $G$ is super connected. Usually, we call a super vertex-cut and the super connectivity for a 1-super vertex-cut and the 1-super connectivity, respectively.

We can similarly define an $h$-super edge-cut and the $h$-super edge-connectivity $\lambda_{h}(G)$ for a (strongly) connected (di)graph $G$. It is also clear that if $\lambda_{1}(G)>\lambda(G)$, then $G$ is super edge-connected. A (di)graph $G$ is said to be optimally super edge-connected if $\lambda_{1}(G)$ exists and there is a minimum super edge-cut $F$ such that $G-F$ has exactly two (strongly) connected components.

For $\lambda_{1}$, it has been extensively studied, (see, for example, $[4,9,11,15]$ ), and it is termed the restricted edge-connectivity denoted by the notation $\lambda^{\prime}$. For $\kappa_{h}$, Esfahanian ${ }^{[3]}$ determined $\kappa_{1}\left(Q_{n}\right)=2 n-2$, and this was further generalized by Latifi et al. ${ }^{[8]}$ to $\kappa_{h}\left(Q_{n}\right)=(n-h) 2^{h}$ for $n \geq 3$ with $0 \leq h \leq\left\lfloor\frac{n}{2}\right\rfloor$, where $Q_{n}$ is the $n$-cube. Up to now, however, we have not seen any results on $\kappa_{1}$ for digraphs in the literature.

In this paper, we consider the relationship between the $h$-super edge-connectivity of a graph and the super connectivity of its line graph. To be precise, we show that for a connected balanced digraph $D$ and its line digraph $L$, if $D$ is optimally super edge-connected, then $\kappa_{1}(L)=2 \lambda_{1}(D)$, and that for a connected graph $G$ and its line graph $L$, if one of $\kappa_{1}(L)$ and $\lambda_{2}(G)$ exists, then $\kappa_{1}(L)=\lambda_{2}(G)$. We determine that $\kappa_{1}(B(d, n))$ is equal to $4 d-8$ for $n=2$ and $d \geq 4$, and to $4 d-4$ for $n \geq 3$ and $d \geq 3$, and that $\kappa_{1}(K(d, n))$ is equal to $4 d-4$ for $d \geq 2$ and $n \geq 2$ except $K(2,2)$. As consequences, we show that $B(d, n)$ and $K(d, n)$ are both super connected for any $d \geq 2$ and $n \geq 1$.

\section{Line Digraphs}

We follow ${ }^{[13]}$ for graph-theoretical terminologies and notations unless otherwise stated. Let $D=(V, E)$ be a strongly connected digraph, in which parallel edges are not allowed. The line digraph of $D$, denoted by $L(D)$, or $L$ for short, is a digraph with vertex set $V(L)=E(D)$, and a vertex $(x, y)$ is adjacent to a vertex $(w, z)$ in $L$ if and only if $y=w$ in $D$. Many properties of line digraphs can be found in [12], one of which is the following lemma.

Lemma 2.1. Let $D$ be a digraph with order at least two. Then $D$ is strongly connected if and only if the line digraph $L(D)$ is strongly connected.

For a subset $E^{\prime} \subseteq E(D)$, we use $D\left[E^{\prime}\right]$ to denote the edge-induced subgraph of $D$ by $E^{\prime}$. Let $L_{1}$ be a subgraph of $L(D)$ and $E_{1}=V\left(L_{1}\right)$. Define $D_{1}=D\left[E_{1}\right]$.

Lemma 2.2. Using the above notations, we have that if $L_{1}$ is a strongly connected subgraph of $L$ with at least two vertices, then the subgraph $D_{1} \subseteq D$ is strongly connected.

Proof. Assume that $x$ and $y$ are any two vertices of $D_{1}$. There is an edge $e$ of $D_{1}$ such that $x$ is incident with the edge $e$. Without loss of generality, we can denote the edge $e$ by $(x, z)$. If $z=y$, then $x$ can reach $y$ by the edge $e$. If $z \neq y$, then $y$ is incident with another edge $e^{\prime}$. Without loss of generality, we can assume the edge $e^{\prime}=(w, y)$. So, $e=(x, z)$ and $e^{\prime}=(w, y)$ are two vertices in $L_{1}$. Since $L_{1}$ is strongly connected, there is a directed path in $L_{1}$ from $(x, z)$ to $(w, y)$ : $\left((x, z),\left(z, z_{1}\right),\left(z_{1}, z_{2}\right), \cdots,\left(z_{k}, w\right),(w, y)\right)$. The corresponding edges $(x, z),\left(z, z_{1}\right),\left(z_{1}, z_{2}\right), \cdots,\left(z_{k}, w\right),(w, y)$ form a directed walk in $D_{1}$ from $x$ to $y$ : $\left(x, z, z_{1}, z_{2}, \cdots, z_{k}, w, y\right)$. So $x$ can reach $y$ in $D_{1}$.

On the other hand, there is also a directed path in $L_{1}$ from $(w, y)$ to $(x, z):((w, y)$, 
$\left.\left(y, y_{1}\right),\left(y_{1}, y_{2}\right), \cdots,\left(y_{l}, x\right),(x, z)\right)$, from which we can construct a directed walk in $D_{1}$ from $y$ to $x$ : $\left(y, y_{1}, y_{2}, \cdots, y_{l}, x\right)$. So $y$ can reach $x$. Therefore, $x$ and $y$ are strongly connected. So, $D_{1}$ is strongly connected.

A digraph $D$ is called balanced if the out-degree $d_{D}^{+}(x)$ is equal to the in-degree $d_{D}^{-}(x)$ for any vertex $x$ of $D$. The following property of balanced digraphs is useful, and the proof of which is simple; see Example 1.4.1 in [13].

Lemma 2.3. If $D$ is a balanced digraph then $|E(X, Y)|=|E(Y, X)|$ for any non-empty proper subsets $X$ and $Y=V(D) \backslash X$, where $E(X, Y)$ denotes the set of edges from $X$ to $Y$ in $D$.

Theorem 2.4. $\quad$ Let $D$ be a connected and balanced digraph and $L=L(D)$.

(a) If $\kappa_{1}(L)$ exists, then $\lambda_{1}(D) \leq \frac{1}{2} \kappa_{1}(L)$.

(b) If $D$ is optimally super edge-connected, then $\kappa_{1}(L)=2 \lambda_{1}(D)$.

Proof. We first note that $D$ is strongly connected by Lemma 2.3.

(a) Assume $\kappa_{1}(L)$ exists and that $E_{0}$ is a super vertex-cut of $L$ with $\left|E_{0}\right|=\kappa_{1}(L)$. Then $L-E_{0}$ is partitioned into several strongly connected components $L_{1}, L_{2}, \cdots, L_{t}$ with $\left|V\left(L_{i}\right)\right| \geq 2$ for $i=1,2, \cdots, t$. Let $D_{i}$ be the edge-induced subgraph of $D$ by $E_{i}=V\left(L_{i}\right)$ and $V_{i}=V\left(D_{i}\right)$ for $i=1,2, \cdots, t$. So $V_{i}$ is nonempty clearly. By Lemma 2.2, $D_{i}$ is strongly connected. Since $\left|E_{i}\right|=\left|V\left(L_{i}\right)\right| \geq 2$ and there is at most one loop at every vertex in $D_{i},\left|V_{i}\right| \geq 2$ for $i=1,2, \cdots, t$.

We claim $V_{i} \cap V_{j}=\emptyset$ for $i, j \in\{1,2, \cdots, t\}$ and $i \neq j$. Suppose to the contrary that $y \in V_{i} \cap V_{j}$ for some $i, j \in\{1,2, \cdots, t\}$ and $i \neq j$. Since $D_{i}$ is strongly connected and $\left|V_{i}\right| \geq 2$, there are vertices $x, z \in V_{i}$ such that $(x, y),(y, z) \in E\left(D_{i}\right)$ (maybe $x=z$ ). Then $(x, y)$ and $(y, z)$, as vertices, are in $L_{i}$. Similarly, there are vertices $x^{\prime}, z^{\prime} \in V_{j}$ such that $\left(x^{\prime}, y\right),\left(y, z^{\prime}\right) \in E\left(D_{j}\right)$ (maybe $x^{\prime}=z^{\prime}$ ). Then $\left(x^{\prime}, y\right)$ and $\left(y, z^{\prime}\right)$, as vertices, are in $L_{j}$. By the construction of $L$, there is an edge $\left((x, y),\left(y, z^{\prime}\right)\right)$ from $V\left(L_{i}\right)$ to $V\left(L_{j}\right)$ and an edge $\left(\left(x^{\prime}, y\right),(y, z)\right)$ from $V\left(L_{j}\right)$ to $V\left(L_{i}\right)$. So, $L_{i} \cup L_{j}$ is strongly connected, a contradiction. Therefore, $V_{i} \cap V_{j}=\emptyset$ for any $i, j \in\{1,2, \cdots, t\}$ and $i \neq j$.

Let $\bar{V}_{1}=V(D) \backslash V_{1}$. Then the edge-sets $E\left(V_{1}, \bar{V}_{1}\right)$ and $E\left(\bar{V}_{1}, V_{1}\right)$ are both super edge-cuts in $D$ and hence $\lambda_{1}(D)$ exists.

To prove $2 \lambda_{1}(D) \leq \kappa_{1}(L)$, we first show $E\left(V_{1}, \bar{V}_{1}\right) \cup E\left(\bar{V}_{1}, V_{1}\right) \subseteq E_{0}$. Suppose to the contrary that there is an edge $(x, y) \in E\left(V_{1}, \bar{V}_{1}\right) \cup E\left(\bar{V}_{1}, V_{1}\right)$ but $(x, y) \notin E_{0}$. Without loss of generality, we suppose $(x, y) \in E\left(V_{1}, \bar{V}_{1}\right)$. So as a vertex of $L,(x, y) \in E-E_{0}$. But $E_{0}$ is a minimum super vertex-cut of $L$, so the vertex $(x, y)$ is in and only in one of the $t$ strongly connected components of $L-E_{0}$. Without loss of generality, assume that $(x, y)$ is in $L_{1}$, then by the definition of $D_{1}$, the edge $(x, y)$ is in $D_{1}$, contradicting to our assumption that $(x, y) \in E\left(V_{1}, \bar{V}_{1}\right)$. So $E\left(V_{1}, \bar{V}_{1}\right) \cup E\left(\bar{V}_{1}, V_{1}\right) \subseteq E_{0}$.

Since $D$ is balanced, $\left|E\left(V_{1}, \bar{V}_{1}\right)\right|=\left|E\left(\bar{V}_{1}, V_{1}\right)\right|$ by Lemma 2.3. So we have

$$
2 \lambda_{1}(D) \leq 2\left|E\left(V_{1}, \bar{V}_{1}\right)\right|=\left|E\left(V_{1}, \bar{V}_{1}\right) \cup E\left(\bar{V}_{1}, V_{1}\right)\right| \leq\left|E_{0}\right|=\kappa_{1}(L) .
$$

(b) Assume that $D$ is optimally super edge-connected. Then $\lambda_{1}(D)$ exists and there is a super edge-cut $F$ of $D$ with $|F|=\lambda_{1}(D)$ such that $G-F$ has exactly two strongly connected components, say $D_{1}$ and $D_{2}$. Let $X=V\left(D_{1}\right)$ and $Y=V\left(D_{2}\right)$. Then $|X| \geq 2$ and $|Y| \geq 2$. Without loss of generality, assume $F=E(X, Y)$. Since $D$ is a connected balanced digraph, $E(Y, X)$ is also a super edge-cut and $|E(Y, X)|=|F|=|E(X, Y)|$ by Lemma 2.3. By Lemma 2.1, $L\left(D_{1}\right)$ and $L\left(D_{2}\right)$ are strongly connected and disjoint. Since $D_{1}$ and $D_{2}$ are strongly connected with at least two vertices, $D_{1}$ and $D_{2}$ both have at least two edges, which implies that $L\left(D_{1}\right)$ and $L\left(D_{2}\right)$ both contain at least two vertices. So $E(X, Y) \cup E(Y, X)$ is a super vertex-cut of $L$. It follows that $\kappa_{1}(L)$ exists and

$$
\kappa_{1}(L) \leq|E(X, Y) \cup E(Y, X)|=2|E(X, Y)|=2 \lambda_{1}(D) .
$$


Combining (1) and (2) yields $\kappa_{1}(L)=2 \lambda_{1}(D)$. The theorem then follows.

\section{3 de Bruijn and Kautz Digraphs}

For an integer $n$, the $n$-th iterated line digraph of $D$ is recursively defined as $L^{n}(D)=$ $L\left(L^{n-1}(D)\right)$ with $L^{0}(D)=D$. In the design of communication networks, line digraphs of some special digraphs are often used as the topology, for they meet many requirements such as small delays and high reliability [12]. The well-known de Bruijn networks and Kautz networks are two of such examples.

For any integers $d \geq 2$ and $n \geq 1$, the de Bruijn digraph $B(d, n)$ can be defined by the $(n-1)$ th iterated line digraph of $K_{d}^{+}$, where $K_{d}^{+}$is a complete digraph of order $d$ plus a loop at every vertex, that is, $B(d, 1)=K_{d}^{+}$and $B(d, n)=L^{n-1}\left(K_{d}^{+}\right)=L(B(d, n-1))$; the Kautz digraph $K(d, n)$ can be defined by the $(n-1)$-th iterated line digraph of $K_{d+1}$, where $K_{d+1}$ is a complete digraph of order $d+1$, that is, $K(d, 1)=K_{d+1}$ and $K(d, n)=L^{n-1}\left(K_{d+1}\right)=L(K(d, n-1))$. In this section, we will determine the super connectivity $\kappa_{1}$ of $B(d, n)$ and $K(d, n)$.

Lemma 3.1 ${ }^{[14]}$. The removal of the efhes incident with the two end-vertices of a pair of symmetric edges of $B(d, n)$ or $K(d, n)$ results in exactly two strongly connected components.

Lemma 3.2 ${ }^{[14]}$. For any de Bruijn digraph $B(d, n)$ with $n \geq 1$ and $d \geq 2$,

$$
\lambda_{1}(B(d, n))= \begin{cases}\infty, & \text { for } n=1 \text { and } 2 \leq d \leq 3, \text { or } \quad n=d=2 ; \\ 2 d-4, & \text { for } n=1 \text { and } d \geq 4 \\ 2 d-2, & \text { otherwise. }\end{cases}
$$

Theorem 3.3. For the de Bruijn digraph $B(d, n)$ with $n \geq 1$ and $d \geq 2$,

$$
\kappa_{1}(B(d, n))=\left\{\begin{aligned}
& \infty, \text { for } n=1, \text { or } \\
& n=2 \text { and } 2 \leq d \leq 3, \text { or } \\
& n=3 \text { and } d=2 ; \\
& 4 d-8, \text { for } n=2 \text { and } d \geq 4 \\
& 4 d-4, \text { otherwise. }
\end{aligned}\right.
$$

Proof. Note that $B(d, n)=L(B(d, n-1))$. It is easy to check that $\kappa_{1}(B(d, n))$ does not exist when $d$ and $n$ take some small values. Combining Theorem 2.4 and Lemma 3.2, we can immediately obtain the theorem if we can prove that $B(d, n)$ is optimally super edge-connected. To this end, by Lemma 3.2, we only need to show that there is a minimum super edge-cut $F$ such that $B(d, n)-F$ contains exactly two strongly connected components when $\lambda_{1}(B(d, n))$ exists. Choose a pair of symmetric edges with end-vertices $\{x, y\}$. Let $X=\{x, y\}, \bar{X}=V(B(d, n)) \backslash X$ and $F=E(X, \bar{X})$. Then $B(d, n)-X$ contains exactly two strongly connected components by Lemma 3.1. Thus, we only need to check that $|F|=\lambda_{1}(B(d, n))$.

When $n=1, B(d, 1)=K_{d}^{+}$. Thus, when $d \geq 4,|F|=|E(X, \bar{X})|=2 d-4=\lambda_{1}(B(d, 1))$ since there are loops at $x$ and $y$ respectively.

When $n \geq 2$ and $d \geq 3$, since there are no loops at $x$ and $y,|F|=|E(X, \bar{X})|=2 d-2=$ $\lambda_{1}(B(d, n))$.

Corollary 3.4. The de Bruijn digraph $B(d, n)$ is super connected for any $d \geq 2$ and $n \geq 1$.

Proof. Since $B(d, 1)$ is a complete digraph of order $d$ with a loop at every vertex, it is clear that $B(d, 1)$ is super connected for any $d \geq 2$. It is easy to see that $B(2,2), B(3,2)$ and $B(2,3)$ 
are super connected. By Theorem 3.3, for $d \geq 4$ and $n=2, \kappa_{1}(B(d, 2))=4 d-8>d-1=$ $\kappa(B(d, n))$, which means that $B(d, 2)$ is super connected for $d \geq 4$. Similarly, for $d \geq 2$ and $n \geq 3$ except $B(2,3), \kappa_{1}(B(d, n))=4 d-4>d-1=\kappa(B(d, n))$, which means that $B(d, n)$ is super connected for $d \geq 2$ and $n \geq 3$.

Lemma 3.5 ${ }^{[14]}$. For any Kautz digraph $K(d, n)$ with $d \geq 2$ and $n \geq 1, \lambda_{1}(K(d, n))=2 d-2$ except $K(2,1)$.

Note that $K(d, n)=L(K(d, n-1))$. Combining Theorem 2.4, Lemma 3.1 and Lemma 3.5, we immediately obtain a similar result for $K(d, n)$.

Theorem 3.6. $\quad \kappa_{1}(K(d, n))=4 d-4$ for $d \geq 2, n \geq 2$ except $K(2,2)$.

Corollary 3.7. The Kautz digraph $K(d, n)$ is super connected for any $d \geq 2$ and $n \geq 1$.

\section{Line Graphs}

Let $G=(V, E)$ be a simple undirected graph. The line graph of $G$, denoted by $L(G)$, or $L$ for short, is an undirected graph, in which $V(L(G))=E(G)$ and two distinct vertices are linked by an edge if and only if they are adjacent as edges of $G$.

It is not always true that for a strongly connected digraph $G$, if $\lambda_{h}(G)$ exists, then there is a minimum super edge-cut $F$ of $G$ such that $G-F$ contains exactly two strongly connected components, but it is not hard to see that for an undirected graph, the following result holds.

Lemma 4.1. For a connected graph $G$, if $\lambda_{h}(G)$ exists, then for any minimum $h$-super edgecut of $G, G-F$ contains exactly two connected components, whereby, it is optimally h-super edge-connected.

Clearly, Lemma 2.1 and Lemma 2.2 are valid for an undirected graph. By Lemma 4.1, if $\lambda_{2}(G)$ exists and $F$ is a minimum 2-super edge-cut of $G$, then the two connected components of $G-F$ both contain at least three vertices, which implies each connected component contains at least two edges. So their line graphs are connected and both contain at least two vertices. Therefore, $L(F)$ corresponds to a super vertex-cut of $L(G)$. If $L_{1}$ is a subgraph of $L(G)$ and connected, then the edge-induced subgraph $G\left[E_{1}\right]$ of $G$ is connected too, where $E_{1}=V\left(L_{1}\right)$. In the same manner as the proof of the digraph case, the following theorem can be obtained, and the detailed proof is omitted here.

Theorem 4.2. If $G$ is a connected undirected graph without parallel edges or loops, then $\kappa_{1}(L)$ exists if and only if $\lambda_{2}(G)$ exists. Moreover, if one of $\lambda_{2}(G)$ and $\kappa_{1}(L)$ exists, then $\kappa_{1}(L)=\lambda_{2}(G)$.

\section{References}

[1] Bauer, D., Boesch, F., Suffel, C., Tindell, R. Connectivity extremal problems and the design of reliable probabilistic networks. The Theory and Application of Graphs. Wiley, New York, 1981, 45-54

[2] Boesch, F.T. Synthesis of reliable networks-A survey. IEEE Trans. Reliability, 35: 240-246 (1986)

[3] Esfahanian, A.H. Generalized measures of fault tolerance with application to $n$-cube networks. IEEE Trans. Comput., 38(11): 1586-1591 (1989)

[4] Esfahanian, A.H., Hakimi, S.L. On computing a conditional edge-connectivity of a graph. Information Processing Letters, 27: 195-199 (1988)

[5] Fábrega, J., Fiol, M.A. Maximally connected digraphs. J. Graph Theory, 13: 657-668 (1989)

[6] Fiol, M.A., Fábrega, J., Escudero, M. Short paths and connectivity in graphs and digraphs. Ars Combin., 29B: 253-256 (1990)

[7] Imase, M., Soneoka, T., Okada, K. Connectivity of regular digraphs with small diameters. IEEE Trans. Comput., 34: 267-273 (1985)

[8] Latifi, S., Hegde, M., Naraghi-Pour, M. Conditional connectivity measures for large miltiprocessor systems. IEEE Trans. Comput., 43: 218-221 (1994) 
[9] Meng, J.X., Ji, Y.H. On a kind of restricted edge connectivity of graphs. Discrete Applied Math., 117: $183-193(2002)$

[10] Soneoka, T. Super edge-connectivity of dense digraphs and graphs. Discrete Applied. Math., 37/38: $511-523(1992)$

[11] Wang, M., Li, Q. Conditional edge connectivity properties, reliability comparisons and transitivity of graphs. Discrete Math., 258: 205-214 (2002)

[12] Xu, J.M. Topological structure and analysis of interconnection networks. Kluwer Academic Publishers, Dordrecht, Boston, London, 2001

[13] Xu, J.M. Theory and application of graphs. Kluwer Academic Publishers, Dordrecht, Boston, London, 2003

[14] Xu, J.M., Lü, M. The restricted edge-connectivity of de Bruijn digraphs. Ars Combinatoria, 75: 490-498 (2005)

[15] Xu, J.M., Xu, K.L. On restricted edge-connectivity of graphs. Discrete Math., 243: 291-298 (2002) 